the North and South poles, the round-trip travel time of the two counter-rotating Brillouin laser modes is different due to the Earth's rotation, as shown in Fig. 1b. This difference changes the frequency splitting of the Brillouin cavity modes, allowing the conversion of rotation into a frequency beat measurement of only a few hertz. In contrast, orienting the gyroscope's axis east and west along the equator induces no rotation along the gyroscope's axis. The monolithic Brillouin gyroscope has an angle random walk as low as 0.068 $\operatorname{deg} \mathrm{h}^{-1 / 2}$ and bias stability $<3.6 \mathrm{deg}^{-1}$. This is particularly impressive given the fact that the Sagnac shift turns out to be extremely small as it scales down with the microresonator effective area. However, performance is still very far from that of state-of-the-art ring laser and fibre-optic gyroscopes that can now readily achieve a strategic-grade sensitivity as high as $10^{-3}$ $\operatorname{deg} \mathrm{h}^{-1}$ and a bias stability better than $10^{-4}$ $\operatorname{deg} \mathrm{h}^{-1}$ (ref. ${ }^{5}$ ). Nonetheless, there is great interest in chip-based photonic devices as they can potentially provide scalability for manufacturing and also resistance to shock and vibration in harsh environments.

As for any novel work, continued progress is required. In the work of Lai and co-workers, resolution and drift compensation could be further improved as could the fundamental limitations from shot noise, Kerr nonlinearity and Rayleigh scattering ${ }^{12}$. That said, the possibilities for chip-scale photonic gyroscopes are promising. A long road still lies ahead before the detection of the precession and nutation of the Earth's axis, or the measurement of exactly how much the rotation is being slowed down by the Moon and the Sun (about 1 second every 50,000 years) can be done as precisely as with atomic clocks.
Franche-Comté, Besançon, France.

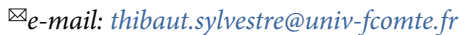

Published online: 29 May 2020

https://doi.org/10.1038/s41566-020-0626-9

References

1. Foucault, L. C. R. Hebd. Séances Acad. Sci. Paris 32, 135-138 (1851).

2. Sagnac, G. C. R. Hebd. Séances Acad. Sci. Paris 95, 708-710 (1913).

3. Michelson, A. A. \& Gale, H. G. J. Astrophys. 61, 401 (1925).

4. Macek, W. M. \& Davis, D. T. M. Jr Appl. Phys. Lett. 2, 67-68 (1963).

5. Lefevre, H. C. The Fiber-Optic Gyroscope 2nd edn (Artech House, 2014).

6. Ciminelli, C., Dell'Olio, F., Campanella, C. E. \& Armenise, M. N. Adv. Opt. Photon. 2, 370-404 (2010)

7. Yang, K. Y. et al. Nat. Photon. 12, 297-302 (2018)

8. Li, J., Suh, M.-G. \& Vahala, K. Optica 4, 346-348 (2017).

9. Liang, W. et al. Optica 4, 114-117 (2017).

10. Lai, Y.-H. et al. Nat. Photon. https://doi.org/10.1038/s41566-0200588-y (2020).

11. Zarinetchi, F., Smith, S. P. \& Ezekiel, S. Opt. Lett. 16 229-231 (1991).

12. Matsko, A. B., Liang, W., Savchenkov, A. A., Ilchenko, V. S. \& Maleki, L. Phys. Lett. A 382, 2289-2295 (2018).

\title{
TERAHERTZ PHOTONICS
}

\section{Cross-nanowire detectors}

Terahertz $(\mathrm{THz})$ waves penetrate through most non-conducting materials, enabling a wide variety of applications including spectroscopy, sensing and imaging. When performing $\mathrm{THz}$ time-domain spectroscopy, fast and precise measurement of the polarization state of $\mathrm{THz}$ waves is desirable. However, currently available polarization-sensitive detectors suffer from crosstalk between detection channels while polarization-insensitive detectors based on wire-grid $\mathrm{THz}$ polarizers require double the data acquisition time. To overcome these technological limits, Kun Peng and co-workers from the UK and Australia have now developed a polarization-sensitive cross-nanowire $\mathrm{THz}$ detector (pictured) that can record the full polarization state of a $\mathrm{THz}$ pulse in one shot without crosstalk (Science 368, 510-513; 2020). The team demonstrated the capabilities of the detector by characterizing the behaviour of a variety of metamaterials.

The cross-nanowire detector is composed of two pairs of gold bowtie electrodes that are bridged by InP nanowires that cross in an orthogonal manner, in order to create two simultaneous and independent measurement channels. The approximate average diameter and length of the nanowires is $280 \mathrm{~nm}$ and $10 \mu \mathrm{m}$,

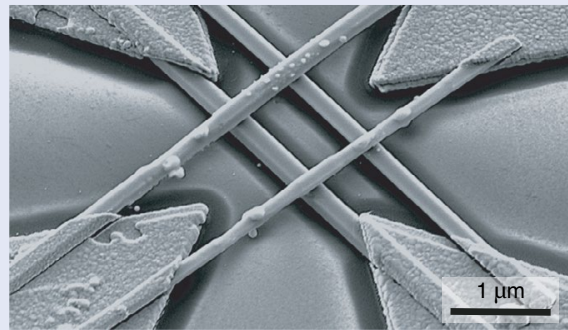

Credit: AAAS

respectively. The nanowires on each bowtie are arranged to be parallel to the gap orientation, and thus the nanowires contacted by different bowtie electrodes are orthogonal while being spatially separated from the substrate to ensure that they are electrically isolated. The cross-nanowire devices were fabricated by two steps of electron-beam lithography and nanowire micropositioning using a transfer print technique to effectively manipulate the nanowire location and orientation in the device.

During a measurement, an incident $\mathrm{THz}$ pulse induces a transient photocurrent in each channel of the detector with the strength of each dictated by the $\mathrm{THz}$ polarization. The response current is maximum when the $\mathrm{THz}$ pulse is polarized parallel to the channel orientation and drops to nearly zero when polarized perpendicular to the channel orientation. Assessment of the polarization sensitivity of the cross-nanowire devices indicates that after calibration a minimum detectable change of polarization angle of $0.38^{\circ}$ is possible.

The international team further investigated the versatility of the cross-nanowire devices: they fabricated metamaterials from twisted split-ring resonator pairs that function as a $\mathrm{THz}$ polarization converter and measured their transmission spectra. Recorded transmission spectra showed good agreement between the simulation and measurement.

The cross-nanowire devices could pave the way to high-speed and high-accuracy $\mathrm{THz}$ pulsed imaging, sensing and spectroscopy. Advantageously, they can replace conventional photoconductive receivers in most $\mathrm{THz}$ time-domain spectroscopy systems without requiring any changes to the optical layout.

Noriaki Horiuchi

Published online: 29 May 2020

https://doi.org/10.1038/s41566-020-0646-5 